УДК $519.16+519.85$

\title{
АППРОКСИМАЦИОННАЯ СХЕМА ХАЙМОВИЧА - РИННОЯ КАНА ДЛЯ CVRР В МЕТРИЧЕСКИХ ПРОСТРАНСТВАХ ФИКСИРОВАННОЙ РАЗМЕРНОСТИ УДВОЕНИЯ ${ }^{1}$
}

\author{
М. Ю. Хачай, Ю. Ю. Огородников
}

\begin{abstract}
Задача маршрутизации транспорта с ограничением на грузоподъемность (Capacitated Vehicle Routing Problem, CVRP) - одна из классических маршрутных экстремальных комбинаторных задач, обладающих большим числом приложений в области исследования операций. Оставаясь NP-трудной в сильном смысле как в общем случае, так и на евклидовой плоскости, задача CVRP допускает квазиполиномиальные и даже полиномиальные приближенные схемы (QPTAS и PTAS) в евклидовых пространствах фиксированной размерности. В то же время метрическая постановка задачи AРХ-полна даже в случае произвольной фиксированной грузоподъемности $q \geq 3$. В данной работе показывается, что классический алгоритм М. Хаймовича и А. Ринноя Кана реализует полиномиальную приближенную схему PTAS и эффективную полиномиальную приближенную схему (EPTAS) в произвольном метрическом пространстве фиксированной размерности при $q=o(\log \log n)$ и произвольной постоянной грузоподъемности соответственно.
\end{abstract}

Ключевые слова: задача маршрутизации транспорта с ограничением на грузоподъемность (CVRP), полиномиально приближенная схема (PTAS), метрическое пространство, размерность удвоения.

M. Yu. Khachai, Yu. Yu. Ogorodnikov. Haimovich-Rinnooy Kan polynomial-time approximation scheme for the CVRP in metric spaces of a fixed doubling dimension.

The Capacitated Vehicle Routing Problem (CVRP) is a classical extremal combinatorial routing problem with numerous applications in operations research. Although the CVRP is strongly NP-hard both in the general case and in the Euclidean plane, it admits quasipolynomial- and even polynomial-time approximation schemes (QPTAS and PTAS) in Euclidean spaces of fixed dimension. At the same time, the metric setting of the problem is APX-complete even for an arbitrary fixed capacity $q \geq 3$. In this paper, we show that the classical HaimovichRinnooy Kan algorithm implements a PTAS and an Efficient Polynomial-Time Approximation Scheme (EPTAS) in an arbitrary metric space of fixed doubling dimension for $q=o(\log \log n)$ and for an arbitrary constant capacity, respectively.

Keywords: Capacitated Vehicle Routing Problem (CVRP), Polynomial-Time Approximation Scheme (PTAS), metric space, doubling dimension.

MSC: 90C27, 90C59, 90B06

DOI: $10.21538 / 0134-4889-2019-25-4-235-248$

\section{Введение}

Задача маршрутизации с ограничением на грузоподъемность транспортных средств (Сараcitated Vehicle Routing Problem, CVRP) - одна из наиболее известных экстремальных комбинаторных задач, обладающая широким спектром приложений в области исследования операций $[11 ; 27]$. По-видимому первая постановка задачи CVRP была приведена Г. Данцигом и Дж. Рамсером в их классической работе [9], посвященной построению экономически эффективного набора маршрутов, обеспечивающих доставку топлива с центрального терминала по сети заправочных станций, распределенной на местности.

Как и для большинства задач комбинаторной оптимизации, результаты в области алгоритмического анализа задачи CVRP условно могут быть разделены на три основных направления.

Первое направление связано с редукцией исследуемой задачи к подходящей постановке задачи целочисленной (смешанной) оптимизации и применением для поиска оптимального

\footnotetext{
${ }^{1}$ Работа выполнена при поддержке РФФИ (проекты 19-07-01243 и 17-08-01385).
} 
решения последней одной из модификаций метода ветвей и границ (см. обзор в [27]). K сожалению, ввиду известной NP-трудности задачи CVRP размеры постановок, поддающихся эффективному решению в рамках данного подхода, остаются достаточно скромными, несмотря на стремительные темпы развития вычислительной техники и очевидные успехи последних лет [22-24] в области совершенствования алгоритмов.

Второе направление связано с применением специализированных эвристик и метаэвристик, среди которых: методы локального поиска [3], переменных окрестностей (VNS) [25], поиска с запретами [26], генетические [28] и биоинспирированные алгоритмы [20], а также их комбинации [19]. Методы этой группы зачастую демонстрируют потрясающую производительность, позволяя эффективно находить близкие к оптимальным или даже точные решения для постановок CVRP чрезвычайно большого размера. Тем не менее отсутствие теоретически обоснованных оценок точности и трудоемкости влечет дополнительные трудозатраты, связанные с численным оцениванием производительности (и возможной донастройкой параметров) алгоритмов при переходе к каждому новому классу постановок. Кроме того, нередко необходимые для этого тестовые наборы данных не могут быть предоставлены заказчиком, например, из соображений безопасности.

Последнее подтверждает актуальность развития третьего направления, связанного с аппроксимируемостью задачи в классе эффективных алгоритмов с гарантированными оценками. Являясь обобщением классической задачи коммивояжера, задача CVRP NP-трудна в сильном смысле и сохраняет труднорешаемость (при условии, что грузоподъемность является частью входа) даже на евклидовой плоскости [21]. Метрическая постановка задачи APX-полна ${ }^{2}$ [5;12], более того, CVRP APX-трудна даже при произвольной фиксированной грузоподъемности $q \geq 3$ и метрики со значениями 0,1 и 2 .

Наибольших успехов в области аппроксимируемости задачи CVRP удалось достичь в конечномерных числовых пространствах. Известные результаты в этой области восходят к классическим работам М. Хаймовича, А. Ринноя Кана [12] и С. Ароры [4]. Так, в статье [12] предложена первая полиномиальная приближенная схема (PTAS) для планарной задачи CVRP при $q=o(\log \log n)$, которую впоследствии удалось распространить на случаи более слабых верхних оценок грузоподъемности (см., например, $[5 ; 16])$, произвольной фиксированной размерности $[17 ; 18]$, дополнительных ограничений на временные промежутки обслуживания [14] и неоднородность спроса [15]. Результат работы [4] - PTAS для евклидовой задачи коммивояжера - лег в основу полиномиальной приближенной схемы [2], находящей $(1+\varepsilon)$-приближенное решение для задачи CVRP на плоскости при рекордно слабой верхней оценке $q \leq 2^{\log ^{\delta(\varepsilon)} n}$, и квазиполиномиальной приближенной схемы (QPTAS) [10] для наиболее общей постановки планарной CVRP в условиях отсутствия каких-либо дополнительных ограничений на грузоподъемность.

Таким образом, известные классы эффективно аппроксимируемых случаев задачи CVRP за редким исключением, быть может, случаев описанных в работе [8], исчерпываются сугубо геометрическими постановками задачи. В то же время, как показано в недавней работе Я. Бартала и коллег [7], близкая к CVRP задача коммивояжера обладает PTAS в существенно более общем случае - в произвольном метрическом пространстве конечной размерности удвоения (doubling dimension).

По-видимому, в данной работе впервые удалось получить аналогичный результат для задачи CVRP.

Мы показываем, что в классе постановок CVRP, задаваемых метрикой размерности удвоения $d>2$ такой, что расстояние $\rho(x, y)$ от произвольного потребителя $x$ до склада $y$ удовлетворяет соотношению $a \leq \rho(x, y) \leq b$ для наперед заданных вещественных чисел $0<a<b$, приближенный алгоритм Хаймовича - Ринноя Кана сохраняет свойства, обоснованные ранее в классической работе [12] для случая евклидовой плоскости. А именно:

\footnotetext{
${ }^{2}$ Т. е. задача аппроксимируема в классе полиномиальных приближенных алгоритмов с фиксированной точностью, и построение для нее полиномиальной приближенной схемы влечет $P=N P$.
} 
1) реализует полиномиальную приближенную схему (PTAS) при условии $q=o(\log \log n)$,

2) является эффективной полиномиальной приближенной схемой (EPTAS) при произвольной фиксированной грузоподъемности $q$.

\section{1. Постановка задачи}

Содержательная постановка задачи CVRP задается множеством потребителей $X$, обладающих идентичным объемом спроса на однородную продукцию (без ограничения общности полагаемым единичным), складом $y$, хранящим неограниченный запас этой продукции, и штатом одинаковых транспортных средств, обладающих заданной грузоподъемностью q. Задача состоит в построении набора циклических маршрутов, начинающихся и завершающихся на складе, удовлетворяющих совокупный потребительский спрос и доставляющих минимальные транспортные издержки.

В свою очередь математическая постановка задачи может быть сформулирована следующим образом. Пусть заданы полный взвешенный граф $G=(X \cup\{y\}, E, w)$ и натуральное число $q$. Симметричная весовая функция $w: E \rightarrow \mathbb{R}_{+}$произвольной паре узлов $\{u, v\} \subset$ $X \cup\{y\}$ сопоставляет транспортные издержки $w(u, v)$. Допустимым маршрутом называется произвольный простой цикл $R=y, x_{i_{1}}, \ldots, x_{i_{s}}, y$ в графе $G$, удовлетворяющий ограничению на грузоподъемность $s \leq q$. Как обычно, стоимостъю маршрута $R$ называем величину $w(R)=w\left(y, x_{i_{1}}\right)+w\left(x_{i_{1}}, x_{i_{2}}\right)+\cdots+w\left(x_{i_{s-1}}, x_{i_{s}}\right)+w\left(x_{i_{s}}, y\right)$. Задача CVRP состоит в построении множества допустимых маршрутов $S=\left\{R_{1}, \ldots, R_{l}\right\}$ минимальной суммарной стоимости $w(S)=\sum_{j=1}^{l} w\left(R_{j}\right)$, удовлетворяющих совокупный потребительский спрос.

Если весовая функция $w$ удовлетворяет неравенству треугольника, т. е. для произвольного подмножества $\left\{v_{1}, v_{2}, v_{3}\right\} \subset X \cup\{y\}$ справедливо соотношение $w\left(v_{1}, v_{2}\right) \leq w\left(v_{1}, v_{3}\right)+w\left(v_{3}, v_{2}\right)$, то величину $w(u, v)$ принято называть расстоянием между точками $u$ и $v$, стоимость $w(R)$ произвольного маршрута $R$ - его длиной, а саму задачу - метрической.

В данной работе мы ограничимся рассмотрением исключительно метрических постановок задачи CVRP. Более того, задавшись произвольным натуральным числом $d>2$ и вещественными числами $0<a<b$, мы потребуем, чтобы каждая рассматриваемая постановка задачи обладала следующими свойствами.

С в о й с т в о 1 . Пара $(Z, \rho)$, в которой $Z=X \cup\{y\}$ и $\left.\rho\right|_{E} \equiv w$, задает метрическое пространство фиксированной размерности удвоения $d>2$.

$\mathrm{C}$ в о й с т в о 2. Расстояние $\rho(x, y)$ от произвольного потребителя $x \in X$ до склада $y$ удовлетворяет двустороннему ограничению $a \leq \rho(x, y) \leq b$.

По традиции всюду ниже мы будем использовать обозначения $\operatorname{CVRP}^{*}(X)$ и $\operatorname{TSP}^{*}(X)$ для оптимальных значений постановок задач маршрутизации и коммивояжера, задаваемых множеством потребителей $X$.

\section{2. Метрические пространства фиксированной размерности удвоения}

Нам потребуется несколько определений и вспомогательных результатов, полученных в теории конечных метрических пространств (см., например, [1]).

О п р е д е л е н и е 1 . Метрическое пространство $(Z, \rho)$ имеет размерность удвоения $d$, если для произвольных $z_{0} \in Z$ и $R>0$ найдутся число $M \leq 2^{d}$ и точки $z_{1}, \ldots, z_{M} \in Z$ такие, что метрический шар

$$
B\left(z_{0}, R\right)=\left\{z \in Z: \rho\left(z_{0}, z\right) \leq R\right\} \subset \bigcup_{j=1}^{M} B\left(z_{j}, R / 2\right) .
$$

Нас будут интересовать инъективные отображения (вложения) $f$ заданного метрического пространства $(Z, \rho)$ в конечномерные линейные нормированные пространства. 
О п р е д е л е н и е 2 . Конечное метрическое пространство $(Z, \rho),|Z|=n$ вкладывается в пространство $l_{p}^{D}$ с искажением $L$, если существует билипшицево вложение $f: Z \rightarrow l_{p}^{D}$, для которого соотношение

$$
\rho\left(z_{1}, z_{2}\right) \leq\left\|f\left(z_{1}\right)-f\left(z_{2}\right)\right\|_{p} \leq L \cdot \rho\left(z_{1}, z_{2}\right)
$$

выполнено для произвольных $z_{1}, z_{2} \in Z$.

Как правило, размерность $D$ результирующего пространства и константа Липшица $L$ зависят от мощности исходного пространства $(Z, \rho)$. Однако для метрических пространств $\left(Z, \rho^{\alpha}\right)$, получаемых из пространств фиксированной размерности удвоения $(Z, \rho)$ путем возведения метрики $\rho$ в произвольную степень $\alpha \in(0,1)$, справедлива известная теорема П. Ассуада [6], гарантирующая вложение таких пространств с константным искажением в пространства фиксированной размерности. Для дальнейших рассуждений нам потребуется более современная версия этого результата [1, теорема 17].

Лемма 1. Пусть $(Z, \rho)$ - произвольное метрическое пространство размерности удвоения $d, p>1,0<\alpha<1, \theta>0$ и $2^{192 / \theta} \leq k \leq d$. Существует билипшицево вложение $f: Z \rightarrow l_{p}^{D}$ mакое, чmо

$$
L=O\left(k^{1+\theta} 2^{d /(p k)} /(1-\alpha)\right) \quad u \quad D=O\left(\frac{d 2^{d / k}}{\alpha \theta}\left(1-\frac{\log (1-\alpha)}{\log k}\right)\right) .
$$

В частности, для используемых нами конкретных значений параметров $p=2, \alpha=1 / 2$, $\theta=192 / \log \log d$ и $k=d$ лемма 1 гарантирует существование вложения $f:(Z, \rho) \rightarrow l_{2}^{D}$ с константой Липшица $L=\operatorname{poly}(d)$ в пространство размерности $D=O(d \log \log d)$.

\section{3. Схема Хаймовича - Ринноя Кана}

В классической статье М. Хаймовича и А. Ринноя Кана [12] предложен приведенный ниже приближенный алгоритм для метрической задачи CVRP и показано, что он реализует полиномиальную приближенную схему (PTAS) для постановок задачи на евклидовой плоскости, удовлетворяющих соотношению $q=o(\log \log n)$.

Алгоритм основан на разбиении множества $X$ на два непересекающихся подмножества $X_{\text {out }}$ и $X_{\text {in }}$ внешних и внутренних потребителей соответственно. Принципиальный момент, на котором базируется обоснование полиномиальности данной схемы, состоит в том, что для поиска $(1+\varepsilon)$-приближенного решения исходной задачи можно ограничиться разбиениями, в которых $\left|X_{\text {out }}\right|$ не зависит от $n$, что позволяет применять для поиска решения соответствующей “внешней" подзадачи точные алгоритмы экспоненциальной трудоемкости. В свою очередь приближенное решение "внутренней" подзадачи, задаваемой подмножеством $X_{i n}$, может быть найдено существенно более грубым, но высокопроизводительным приближенным методом итерированного разбиения маршрута (ITP) [12], принимающим на вход произвольное $\beta$-приближенное решение вспомогательной метрической задачи коммивояжера.

А л г о р и т м Хаймовича - Ринноя Кана

1. Упорядочить множество потребителей $X$ по убыванию расстояния $r_{i}=\rho\left(x_{i}, y\right)$ от склада.

2. Для заданного $\varepsilon>0$ найти наименьшее значение $\tilde{k}=\tilde{k}(\varepsilon, q, \beta)$, для которого относительная погрешность алгоритма удовлетворяет соотношению

$$
e(\tilde{k})=\frac{\operatorname{CVRP}^{*}\left(X_{\text {out }}\right)+\operatorname{ITP}\left(X_{\text {in }}\right)-\operatorname{CVRP}^{*}(X)}{\operatorname{CVRP}^{*}(X)} \leq \varepsilon .
$$

Здесь $X_{\text {out }}=\left\{x_{1}, \ldots, x_{\tilde{k}-1}\right\}, X_{\text {in }}=X \backslash X_{\text {out }}$ и $\operatorname{ITP}\left(X_{\text {in }}\right)$ - верхняя оценка стоимости приближенного решения, возвращаемого алгоритмом ITP. 
3. Для поиска точного решения $\mathrm{S}_{\mathrm{DP}}$ "внешней" подзадачи и приближенного решения $\mathrm{S}_{\mathrm{ITP}}$ "внутренней" применить схему динамического программирования Хорна [13] и алгоритм ITP соответственно.

4. Выдать приближенное решение исходной задачи в виде $S=\mathrm{S}_{\mathrm{DP}} \cup \mathrm{S}_{\mathrm{ITP}}$.

\section{4. Основной результат}

В этом разделе мы покажем, что результат Хаймовича - Ринноя Кана справедлив для гораздо более широкого (чем евклидова плоскость) класса постановок CVRP.

Теорема. $(1+\varepsilon)$-приближенное решение произвольной метрической постановки задачи CVRP, обладающей свойствами 1 и 2 , может быть получено для произвольного $\varepsilon>0$ за время

$$
O\left(q k^{3} 2^{k}\right)+\operatorname{TIME}(\mathrm{TSP}, \beta, n)+O\left(n^{2}\right)
$$

әде

$$
k=k(\varepsilon, q, \beta, D, a)=O\left(\left(\frac{q}{\varepsilon}\right)^{D}\left(\frac{4 \sqrt{2} \beta D^{(1+3 / D) / 2} L}{\sqrt{a}}\right)^{D}+\frac{\beta D^{2} 2^{D} L}{2 \sqrt{a}}\right)\left(\exp \left(\frac{q}{\varepsilon}\right)\right)^{2},
$$

TIME(TSP, $\beta, n)$ - время решения вспомогательной задачи коммивояжера, $D=O(d \log \log d)$ и $L=\operatorname{poly}(d)$.

Д о к а з а т е л ь с т в о теоремы следует из приведенных ниже известных лемм (см., например, $[5 ; 12])$.

Лемма 2. Стоимость $w\left(\mathrm{~S}_{\mathrm{ITP}}\right)$ приближенного решения, получаемого методом ITP для произвольной (необязательно метрической) постановки CVRP, удовлетворяет соотношению

$$
\operatorname{ITP}(X)=w\left(S_{\text {ITP }}\right) \leq 2\left\lceil\frac{n}{q}\right\rceil \frac{\sum_{i=1}^{n} r_{i}}{n}+(1-1 / q) \operatorname{TSP}^{*}(X) .
$$

Лемма 3. Стоимость оптимального решения $\mathrm{CVRP}^{*}(X)$ произвольной метрической постановки задачи CVRP допускает нижнюю оценку

$$
\operatorname{CVRP}^{*}(X) \geq \max \left\{\operatorname{TSP}^{*}(X \cup\{y\}), 2 r_{1}, \frac{2}{q} \sum_{i=1}^{n} r_{i}\right\} .
$$

Следующая лемма устанавливает верхнюю оценку неустранимой погрешности, возникающей на шаге 2 схемы Хаймовича - Ринноя Кана при декомпозиции исходной постановки.

Лемма 4. Для произвольного $1 \leq k \leq n$ и разбиения множества потребителей $X$ произвольной метрической постановки CVRP на подмножества $X_{\text {in }}$ и $X_{\text {оut }}$ справедливо соотношение

$$
\operatorname{CVRP}^{*}\left(X_{\text {in }}\right)+\operatorname{CVRP}^{*}\left(X_{\text {out }}\right) \leq \operatorname{CVRP}^{*}(X)+4(k-1) r_{k} .
$$

Последняя лемма из ранее доказанных устанавливает верхнюю оценку для веса оптимального решения задачи коммивояжера на множестве потребителей $X \in l_{2}^{D}$. Впервые данный технический результат был представлен в работе [17, лемма 5].

Лемма 5. Пусть $X \subset B(0, R)=\left\{x \in l_{2}^{D}:\|x\|_{2} \leq R\right\}$. Для длины $\operatorname{TSP}^{*}(X)$ оптимального маршрута коммивояжера справедлива верхняя оценка

$$
\operatorname{TSP}^{*}(X) \leq C_{D} R+C_{D}^{*} R^{1 / D}\left(\sum_{i=1}^{n} r_{i}\right)^{1-1 / D}
$$

где $C_{D}=D^{2} 2^{D+1}$ u $C_{D}^{*}=4 \sqrt{2} D^{(1+3 / D) / 2}$. 
Д о к а з а т е л ь с т в о. 1. Рассмотрим метрическое пространство $\mathcal{S}=\left(S^{D-1}\right.$, dist $)$, задаваемое на поверхности единичной сферы $S^{D-1}$ пространства $l_{2}^{D}$ угловым расстоянием $\operatorname{dist}\left(\xi_{1}, \xi_{2}\right)=\arccos \left(\xi_{1}, \xi_{2}\right)$. Проверим, что пространство $\mathcal{S}$ содержит конечное $\delta$-плотное подмножество для произвольного достаточно малого $\delta>0$. В самом деле, поместим сферу $S^{D-1}$ в $D$-мерный гиперкуб со стороной 2 . Задавшись произвольным $h \in(0,2]$, разобьем каждое одномерное ребро гиперкуба на $\left\lceil\frac{2}{h}\right\rceil$ ячеек, каждая из которых, за исключением, может быть, одной, имеет длину $h$. В свою очередь каждая фасета гиперкуба будет содержать $\left\lceil\frac{2}{h}\right\rceil^{D-1}$ ячеек размерности $D-1$, а общее число ячеек на его поверхности составит

$$
2 D\lceil 2 / h\rceil^{D-1} \leq 2 D(1+2 / h)^{D-1}=2^{D} D h^{1-D}(1+h / 2)^{D-1} \leq \tilde{C}_{D} h^{1-D},
$$

где $2 \tilde{C}_{D}=D 4^{D}$.

Легко убедиться в том, что множество $\mathcal{S}_{h}^{\prime}$, состоящее из проекций геометрических центров построенных ячеек на поверхность сферы $S^{D-1}$, является искомым $\delta$-плотным подмножеством $\mathcal{S}$ при $\delta=h \sqrt{D-1} / 2$, мощность которого не превосходит $\tilde{C}_{D} h^{1-D}$.

2. Для получения искомой верхней оценки $\operatorname{TSP}^{*}(X)$ сделаем следующее:

- через каждую точку $\xi_{j} \in \mathcal{S}_{h}^{\prime}$ проведем радиус шара $B(0, R)$, содержащего множество $X$;

- произвольную точку $x_{i} \in X$ соединим перпендикуляром с ближайшим к ней радиусом;

- построим замкнутый маршрут, проходящий по каждому из построенных радиусов от начала координат до поверхности шара $B(0, R)$, посещая при необходимости присоединенные к нему точки $x_{i}$.

Легко видеть, что длина построенного маршрута не превосходит величины

$$
W(h)=2 \tilde{C}_{D} h^{1-D} R+h \sqrt{D-1} \sum_{i=1}^{n} r_{i}
$$

и, следовательно,

$$
\operatorname{TSP}^{*}(X) \leq \min \{W(h): 0<h \leq 2\} .
$$

В силу выпуклости функции $W=W(h)$ оптимум в задаче (2) достигается либо в стационарной точке

$$
h^{*}=\left(\frac{2 \tilde{C}_{D} R \sqrt{D-1}}{\sum_{i=1}^{n} r_{i}}\right)^{1 / D}
$$

в которой

$$
W^{\prime}(h)=2 \tilde{C}_{D}(1-D) R h^{-D}+\sqrt{D-1} \sum_{i=1}^{n} r_{i}=0,
$$

либо при $h^{*} \geq 2$ на правой границе интервала.

Рассмотрим каждый из случаев в отдельности. Пусть $h^{*}<2$, тогда

$$
\begin{aligned}
& \operatorname{TSP}^{*} \leq W\left(h^{*}\right)=2 \tilde{C}_{D} R\left(\frac{2 \tilde{C}_{D} R \sqrt{D-1}}{\sum_{i=1}^{n} r_{i}}\right)^{\frac{1-D}{D}}+\left(\frac{2 \tilde{C}_{D} R \sqrt{D-1}}{\sum_{i=1}^{n} r_{i}}\right)^{1 / D} \sqrt{D-1} \sum_{i=1}^{n} r_{i} \\
& =\left(2 \tilde{C}_{D}\right)^{1 / D}\left((D-1)^{\frac{1-D}{2 D}}+(D-1)^{\frac{D+1}{2 D}}\right) R^{1 / D}\left(\sum_{i=1}^{n} r_{i}\right)^{1-1 / D} \leq C_{D}^{*} R^{1 / D}\left(\sum_{i=1}^{n} r_{i}\right)^{1-1 / D}
\end{aligned}
$$

где $4 D^{1 / D}\left((D-1)^{\frac{1-D}{2 D}}+(D-1)^{\frac{D+1}{2 D}}\right) \leq C_{D}^{*}=4 \sqrt{2} D^{(1+3 / D) / 2}$ при произвольном $D>1$. 
Если $h^{*} \geq 2$, то из соотношения (3) имеем $\sum_{i=1}^{n} r_{i} \leq 2^{1-D} \tilde{C}_{D} R \sqrt{D-1}$, откуда

$$
\begin{gathered}
\operatorname{TSP}^{*} \leq W(2)=2 \tilde{C}_{D} 2^{1-D} R+2 \sqrt{D-1} \sum_{i=1}^{n} r_{i} \\
\leq 2^{2-D} \tilde{C}_{D} R+2^{2-D}(D-1) \tilde{C}_{D} R=2^{1-D} D^{2} 2^{2 D} R=D^{2} 2^{D+1} \cdot R .
\end{gathered}
$$

Полагая $C_{D}=D^{2} 2^{D+1}$ и суммируя оценки (4) и (5), завершаем обоснование неравенства (1).

Лемма доказана.

Рассмотрим теперь произвольную метрическую постановку задачи CVRP, обладающую свойствами 1 и 2 . Зададимся произвольным непустым подмножеством $X^{\prime}=X \cap B(y, R)$ множества потребителей, определим $R=\max \left\{r_{i}: x_{i} \in X^{\prime}\right\}$ и получим верхнюю оценку длины $\operatorname{TSP}^{*}\left(X^{\prime}\right)$ оптимального маршрута коммивояжера в терминах расстояний от потребителей до складов, подобную оценке (1). Без ограничения общности полагаем $b \leq 1 / 2$.

Пусть $f: Z \rightarrow l_{2}^{D}$ - вложение пространства $\left(Z, \rho^{1 / 2}\right)$ в конечномерное евклидово пространство, соответствующее конкретным значениям параметров $p=2, \alpha=1 / 2, \theta=192 / \log \log d$ и $k=d$, удовлетворяющее соотношению

$$
\rho^{1 / 2}\left(z_{1}, z_{2}\right) \leq\left\|f\left(z_{1}\right)-f\left(z_{2}\right)\right\|_{2} \leq L \rho^{1 / 2}\left(z_{1}, z_{2}\right) .
$$

Существование вложения $f$ гарантируется леммой 1 для размерности $D=O(d \log \log d)$ и $L=\operatorname{poly}(d)$. По построению неравенство

$$
\rho\left(z_{1}, z_{2}\right) \leq \rho^{1 / 2}\left(z_{1}, z_{2}\right) \leq 1
$$

верно для произвольной пары $\left\{z_{1}, z_{2}\right\}$ из подмножества $Z^{\prime}=X^{\prime} \cup\{y\}$.

Обозначим длину оптимальных маршрутов коммивояжера на множестве $X^{\prime}$ в исходной метрике $\rho$, метрике $\rho^{1 / 2}$ и на множестве $f\left(X^{\prime}\right) \subset l_{2}^{D}$ в метрике, порождаемой евклидовой нормой через $\operatorname{TSP}_{\rho}^{*}\left(X^{\prime}\right), \operatorname{TSP}_{\rho^{1 / 2}}^{*}\left(X^{\prime}\right)$ и $\operatorname{TSP}_{l_{2}^{D}}^{*}\left(X^{\prime}\right)$ соответственно.

Лемма 6. Справедлива следующая оченка:

$$
\operatorname{TSP}_{\rho}^{*}\left(X^{\prime}\right) \leq \operatorname{TSP}_{\rho^{1 / 2}}^{*}\left(X^{\prime}\right) \leq \operatorname{TSP}_{l_{2}^{D}}^{*}\left(X^{\prime}\right) \leq \frac{C_{D} L}{\sqrt{a}} R+\frac{C_{D}^{*} L}{\sqrt{a}} R^{1 / D}\left(\sum_{x_{i} \in X^{\prime}} r_{i}\right)^{1-1 / D}
$$

Д о к а з а т е л ь с т в о. Неравенства $\operatorname{TSP}_{\rho}^{*}\left(X^{\prime}\right) \leq \operatorname{TSP}_{\rho^{1 / 2}}^{*}\left(X^{\prime}\right) \leq \operatorname{TSP}_{l_{2}^{D}}^{*}\left(X^{\prime}\right)$ следуют непосредственно из соотношений (6) и (7). В самом деле, обоснуем, например, первое из них. Пусть $T$ - оптимальный маршрут коммивояжера в метрике $\rho^{1 / 2}$. Неравенство (7) влечет соотношение $w_{\rho}(T) \leq w_{\rho^{1 / 2}}(T)$ для весов маршрута $T$ в исходной метрике и метрике $\rho^{1 / 2}$ соответственно. Следовательно,

$$
\operatorname{TSP}_{\rho}^{*}\left(X^{\prime}\right) \leq w_{\rho}(T) \leq w_{\rho^{1 / 2}}(T)=\operatorname{TSP}_{\rho^{1 / 2}}^{*}\left(X^{\prime}\right)
$$

Далее, по лемме 5

$$
\operatorname{TSP}_{l_{2}^{D}}^{*}\left(X^{\prime}\right) \leq C_{D} \tilde{R}+C_{D}^{*} \tilde{R}^{1 / D}\left(\sum_{x_{i} \in X^{\prime}} \tilde{r}_{i}\right)^{1-1 / D}
$$

где $\tilde{r}_{i}=\left\|f\left(x_{i}\right)-f(y)\right\|_{2}$ и $\tilde{R}=\max \left\{\tilde{r}_{i}: x_{i} \in X^{\prime}\right\}$. Поскольку рассматриваемая постановка обладает свойством 2 , для каждого $x_{i} \in X^{\prime}$ имеем $\sqrt{r_{i}} \leq r_{i} / \sqrt{a}$, и, следовательно, в силу (6) $\tilde{r}_{i} \leq L \sqrt{r_{i}} \leq L / \sqrt{a} r_{i}$. Применяя это соображение к соотношению (8) и учитывая доказанное выше, получаем искомую оценку.

Лемма доказана.

Далее оценим относительную погрешность $e(k)$ исследуемой схемы. 
Лемма 7. Для любого $k<n$ относительная погрешность е $(k)$ может быть оценена следующим образом:

$$
e(k) \leq q\left(2 k+1+\frac{\beta C_{D} L}{2 \sqrt{a}}\right) \frac{r_{k}}{\sum_{i=1}^{n} r_{i}}+\frac{q \beta C_{D}^{*} L}{2 \sqrt{a}}\left(\frac{r_{k}}{\sum_{i=1}^{n} r_{i}}\right)^{1 / D},
$$

где $\beta$ - точность решения вспомогательной задачи коммивояљера.

Д о к а з а т е л ь с т в о. По определению для рассматриваемой схемы $e(k)$ имеет вид

$$
e(k)=\frac{\operatorname{CVRP}^{*}\left(X_{\text {out }}\right)+\operatorname{ITP}\left(X_{\text {in }}\right)-\operatorname{CVRP}^{*}(X)}{\operatorname{CVRP}^{*}(X)} .
$$

Добавим и вычтем в числителе величину $\operatorname{CVRP}^{*}\left(X_{i n}\right)$ :

$$
e(k)=\frac{\operatorname{CVRP}^{*}\left(X_{\text {out }}\right)+\operatorname{ITP}\left(X_{\text {in }}\right)-\operatorname{CVRP}^{*}(X)+\operatorname{CVRP}^{*}\left(X_{\text {in }}\right)-\operatorname{CVRP}^{*}\left(X_{\text {in }}\right)}{\operatorname{CVRP}^{*}(X)} .
$$

Воспользовавшись леммами 2-6, получаем цепочку неравенств

$$
\begin{aligned}
e(k) & \leq \frac{4(k-1) r_{k}+\frac{2}{q} \sum_{i=k}^{n} r_{i}+\beta \operatorname{TSP}^{*}\left(X_{i n}\right)+2 r_{k}-\frac{2}{q} \sum_{i=k}^{n} r_{i}}{\frac{2}{q} \sum_{i=1}^{n} r_{i}} \\
& \leq \frac{4 k r_{k}+\beta\left(\frac{C_{D} L r_{k}}{\sqrt{a}}+\frac{C_{D}^{*} L r_{k}^{1 / D}}{\sqrt{a}}\left(\sum_{i=k}^{n} r_{i}\right)^{1-1 / D}\right)+2 r_{k}}{\frac{2}{q} \sum_{i=1}^{n} r_{i}} \\
& \leq q\left(2 k+1+\frac{\beta C_{D} L}{2 \sqrt{a}}\right) \frac{r_{k}}{\sum_{i=1}^{n} r_{i}}+\frac{q \beta C_{D}^{*} L}{2 \sqrt{a}}\left(\frac{r_{k}}{\sum_{i=1}^{n} r_{i}}\right)^{1 / D} .
\end{aligned}
$$

Лемма доказана.

Доказательство существования для произвольного заданного $\varepsilon>0$ номера $k=k(\varepsilon)$, обеспечивающего верхнюю оценку относительно погрешности $e(k)<\varepsilon$, основано на следующей технической лемме.

Лемма 8. Пусть для некоторых чисел $A, B, C>0$ u $D>1$ система

$$
s_{k}^{D}+\frac{2 B}{2 k+A} s_{k}-\frac{C}{2 k+A} \geq 0 \quad(1 \leq k \leq \tilde{k})
$$

обладает решением $s_{1}, s_{2}, \ldots, s_{\tilde{k}}$, для которого $\sum_{k=1}^{\tilde{k}} s_{k}^{D} \leq 1$. Тогда

$$
\tilde{k} \leq\left(\left(\frac{C}{4 B}\right)^{-D}+A+1\right) \exp \left(\frac{2}{C}\right) .
$$

Д о к а з а т е л ь с т в о. В самом деле, зададимся произвольным решением $s_{1}, s_{2}, \ldots, s_{\tilde{k}}$ системы (9), удовлетворяющим соотношению $\sum_{k=1}^{\tilde{k}} s_{k}^{D} \leq 1$. Для произвольного $1 \leq k \leq \tilde{k}$ возможен один из двух вариантов

$$
s_{k}^{D} \leq \frac{C / 2}{2 k+A} \quad \text { и } \quad s_{k}^{D}>\frac{C / 2}{2 k+A} .
$$


Пусть $K \subset\{1, \ldots, \tilde{k}\}-$ подмножество номеров неравенств, для которых справедлива первая альтернатива. Для каждого $k \in K$ из соответствующего неравенства системы (9) имеем

$$
\frac{2 B}{2 k+A} s_{k} \geq \frac{C / 2}{2 k+A}
$$

т. е. $s_{k}^{D} \geq \frac{C}{4 B}$. Таким образом,

$1 \geq \sum_{k=1}^{\tilde{k}} s_{k}^{D}=\sum_{k \in K} s_{k}^{D}+\sum_{k \notin K} s_{k}^{D} \geq\left(\frac{C}{4 B}\right)^{D}|K|+\frac{C}{2} \sum_{k \notin K} \frac{1}{2 k+A} \geq\left(\frac{C}{4 B}\right)^{D}|K|+\frac{C}{2} \sum_{k=|K|+1}^{\tilde{k}} \frac{1}{2 k+A}$,

где справедливость последнего неравенства следует из монотонности функции $1 /(2 k+A)$. Поскольку для произвольного $|K|<\tilde{k}$

$$
\left(\frac{C}{4 B}\right)^{D}|K|+\int_{|K|+1}^{\tilde{k}} \frac{C d x}{2(x+A)}=\left(\frac{C}{4 B}\right)^{D}|K|+\frac{C}{2} \ln \left(\frac{\tilde{k}+A}{|K|+1+A}\right)
$$

то

$$
1 \geq \max \left\{\frac{C}{2} \ln \left(\frac{\tilde{k}+A}{|K|+1+A}\right),\left(\frac{C}{4 B}\right)^{D}|K|\right\}
$$

откуда

$$
\tilde{k} \leq(|K|+1+A) \exp \left(\frac{2}{C}\right)=\left(\left(\frac{C}{4 B}\right)^{-D}+A+1\right) \exp \left(\frac{2}{C}\right)
$$

Лемма доказана.

Следующая лемма, устанавливающая точность алгоритма Хаймовича - Ринноя Кана, является непосредственным следствием леммы 8.

Лемма 9. Для произвольных значений параметров $\varepsilon>0, \beta \geq 1, D>2, q>1$ существует значение

$$
\tilde{k}=\tilde{k}(\varepsilon, q, \beta, D, a)=O\left(\left(\frac{q}{\varepsilon}\right)^{D}\left(\frac{4 \sqrt{2} \beta D^{(1+3 / D) / 2} L}{\sqrt{a}}\right)^{D}+\frac{\beta D^{2} 2^{D} L}{2 \sqrt{a}}\right)\left(\exp \left(\frac{q}{\varepsilon}\right)\right)^{2}
$$

такое, что условие

$$
e(k) \leq q\left(2 k+1+\frac{\beta C_{D} L}{2 \sqrt{a}}\right) \frac{r_{k}}{\sum_{i=1}^{n} r_{i}}+\frac{q \beta C_{D}^{*} L}{2 \sqrt{a}}\left(\frac{r_{k}}{\sum_{i=1}^{n} r_{i}}\right)^{1 / D}<\varepsilon
$$

выполнено по крайней мере для одного значения $k$ из интервала $1 \leq k \leq \tilde{k}$.

Д о к а з а т е л ь с т в о. В самом деле, достаточно положить

$$
s_{k}=\left(\frac{r_{k}}{\sum_{i=1}^{n} r_{i}}\right)^{1 / D}, \quad A=1+\frac{\beta D^{2} 2^{D} L}{2 \sqrt{a}}, \quad B=\frac{\beta \sqrt{2} D^{(1+3 / D) / 2} L}{\sqrt{a}}, \quad C=\frac{\varepsilon}{q},
$$

после чего применить утверждение леммы 8.

Лемма доказана. 
Для завершения д о к а з а т е л ь с т в а теоремы оценим трудоемкость схемы Хаймовича - Ринноя Кана. В самом деле, трудоемкость поиска оптимального решения "внешней" подзадачи для подмножества потребителей $X_{\text {out }}$ посредством схемы динамического программирования Хорна [13] не превосходит $O\left(q \tilde{k}^{3} 2^{\tilde{k}}\right)$, где верхняя оценка $\tilde{k}$ приведена в лемме 9. Трудоемкость приближенного решения "внутренней" подзадачи определяется трудоемкостью TIME(TSP, $\beta, n) \beta$-приближенного алгоритма для вспомогательной метрической задачи коммивояжера ${ }^{3}$. Учитывая известную верхнюю оценку $O\left(n^{2}\right)$ времени работы алгоритма ITP, получаем суммарную общую оценку

$$
O\left(q \tilde{k}^{3} 2^{\tilde{k}}\right)+\operatorname{TIME}(\mathrm{TSP}, \beta, n)+O\left(n^{2}\right)
$$

трудоемкости исследуемой схемы, чем завершаем доказательство теоремы.

Следствие. Алгоритм Хаймовича - Ринноя Кана в классе метрических постановок CVRP, обладающих свойствами 1 и 2 , реализует полиномиально приблиюсенную схему (PTAS) при $q=o(\log \log n)$ и эффективную полиномиально приближенную схему (EPTAS) для произвольного фиксированного значения $q$.

\section{5. Заключение}

В данной работе впервые показано, что семейство постановок задачи CVRP, аппроксимируемых за полиномиальное время с любой заданной точностью, не ограничено постановками, заданными в конечномерных числовых пространствах. В частности, доказано, что классический алгоритм М. Хаймовича и А. Ринноя Кана сохраняет аппроксимационные свойства, обоснованные ранее для конечномерных евклидовых пространств, в пространствах существенно более общей природы - метрических пространствах с фиксированной размерностью удвоения. Как следует из результатов статьи, данный алгоритм реализует полиномиальную приближенную схему (PTAS) при условии отделимости потребителей от склада и ограничении на грузоподъемность $q=o(\log \log n)$ и является эффективной полиномиальной приближенной схемой (EPTAS) при произвольном фиксированном q. Отметим, что процедура поиска $(1+\varepsilon)$-приближенного решения задачи CVRP реализуется исследуемым в работе алгоритмом непосредственно в исходном метрическом пространстве. Техника билипшицевого вложения метрического пространства в пространство $l_{2}^{D}$ подходящей размерности используется в доказательстве теоремы исключительно при обосновании точности приближения. Из вопросов, оставшихся на данный момент открытыми, отметим возможность распространения результата A. Дас и K. Матье [10] об аппроксимируемости CVRP без ограничения роста грузоподъемности в классе квазиполиномиальных приближенных схем (QPTAS) на семейство метрических постановок в пространствах ограниченной размерности удвоения.

\section{СПИСОК ЛИТЕРАТУРЫ}

1. Abraham I., Bartal Y., Neiman O. Advances in metric embedding theory // Advances in Mathematics. 2011. Vol. 228, no. 6. P. 3026-3126. https://doi.org/10.1016/j.aim.2011.08.003

2. Adamaszek A., Czumaj A., Lingas A. PTAS for $k$-tour cover problem on the plane for moderately large values of $k / /$ Inter. J. of Foundations of Computer Science. 2010. Vol. 21, no. 06. P. 893-904. https://doi.org/10.1142/S0129054110007623

3. Arnold F., Sörensen K. Knowledge-guided local search for the vehicle routing problem // Comput. Oper. Res. 2019. Vol. 105. P. 32-46. https://doi.org/10.1016/j.cor.2019.01.002

4. Arora S. Polynomial time approximation schemes for Euclidean traveling salesman and other geometric problems // J. ACM. 1998. Vol. 45. P. 753-782.

\footnotetext{
${ }^{3}$ Например, для алгоритма Кристофидеса - Сердюкова $\beta=3 / 2$ и $\operatorname{TIME}(\mathrm{TSP}, \beta, n)=O\left(n^{3}\right)$.
} 
5. Asano T., Katoh N., Tamaki H., Tokuyama T. Covering points in the plane by $k$-tours: Towards a polynomial time approximation scheme for general $k / /$ Proceedings of the Twenty-ninth Annual ACM Symposium on Theory of Computing (STOC '97). N Y: ACM, 1997. P. 275-283. https://doi.org/10.1145/258533.258602

6. Assouad P. Plongements lipschitziens dans $\mathbb{R}^{n} / /$ Bulletin de la Société Mathématique de France. 1983. Vol. 111. P. 429-448. http://eudml.org/doc/87452

7. Bartal Y., Gottlieb L. A., Krauthgamer R. The traveling salesman problem: Low-dimensionality implies a polynomial time approximation scheme // SIAM J. Computing. 2016. Vol. 45, no. 4. P. 1563-1581. https://doi.org/10.1137/130913328

8. Becker A., Klein P. N., Schild A. A PTAS for bounded-capacity vehicle routing in planar graphs // Algorithms and Data Structures / eds. Z. Friggstad., J.-R. Sack, M. Salavatipour. Cham: Springer, 2019. P. 99-111. (Lecture Notes in Computer Science; vol. 11646).

9. Dantzig G. B., Ramser J. H. The truck dispatching problem // Management science. 1959. Vol. 6, no. 1. P. $80-91$.

10. Das A., Mathieu C. A quasipolynomial time approximation scheme for Euclidean capacitated vehicle routing // Algorithmica. 2015. Vol. 73. P. 115-142. https://doi.org/10.1007/s00453-014-9906-4

11. Demir E., Huckle K., Syntetos A., Lahy A., Wilson M. Vehicle routing problem: Past and future // Contemporary Operations and Logistics / ed. P. Wells Cham: Springer, 2019. P. 97-117. https://doi.org/10.1007/978-3-030-14493-7_7

12. Haimovich M., Rinnooy Kan A.H. G. Bounds and heuristics for capacitated routing problems // Math. Oper. Res. 1985. Vol. 10, no. 4. P. 527-542. https://doi.org/10.1287/moor.10.4.527

13. van Hoorn J. J. Dynamic programming for routing and scheduling: Optimizing sequences of decisions. Ph. D. thesis, 2016. 210 p. https://doi.org/10.13140/RG.2.2.14344.88329

14. Khachay M., Ogorodnikov Y. Efficient PTAS for the Euclidean CVRP with time windows // Analysis of Images, Social Networks and Texts (AIST 2018) / ed. van der W. Aalst. Cham: Springer, 2018. P. 318-328. (Lecture Notes in Computer Science; vol. 11179).

https://doi.org/10.1007/978-3-030-11027-7_30

15. Khachay M., Ogorodnikov Y. Approximation scheme for the capacitated vehicle routing problem with time windows and non-uniform demand // Mathematical Optimization Theory and Operations Research (MOTOR 2019) / eds. M. Khachay, Y. Kochetov, P. Pardalos. Cham: Springer, 2019. P. 309327. (Lecture Notes in Computer Science; vol. 11548). https://doi.org/10.1007/978-3-030-22629-9_22

16. Khachay M., Ogorodnikov Y. Improved polynomial time approximation scheme for capacitated vehicle routing problem with time windows // Optimization and Applications (OPTIMA 2018) / Y. Evtushenko, M. Jaćimović, M. Khachay, Y. Kochetov, V. Malkova, M. Posypkin. Cham: Springer, 2019. P. 155-169. (Communications in Computer and Information Science; vol. 974). https://doi.org/10.1007/978-3-030-10934-9_12

17. Khachay M., Dubinin R. PTAS for the Euclidean capacitated vehicle routing problem in $R^{d} / /$ Discrete Optimization and Operations Research / eds. Y. Kochetov, M. Khachay, V. Beresnev, E. Nurminski, P. Pardalos. Cham: Springer, 2016. P. 193-205. (Lecture Notes in Computer Science,;vol. 9869). https://doi.org/10.1007/978-3-319-44914-2_16

18. Khachay M., Zaytseva H. Polynomial time approximation scheme for single-depot Euclidean capacitated vehicle routing problem // Combinatorial Optimization and Applications / eds. Z. Lu, D. Kim, W. Wu, W. Li, D.Z Du. Cham: Springer, 2015. P. 178-190. (Lecture Notes in Computer Science; vol. 9486). https://doi.org/10.1007/978-3-319-26626-8_14

19. Nalepa J., Blocho M. Adaptive memetic algorithm for minimizing distance in the vehicle routing problem with time windows // Soft Computing. 2016. Vol. 20, no. 6. P. 2309-2327. https://doi.org/10.1007/s00500-015-1642-4

20. Necula R., Breaban M., Raschip M. Tackling dynamic vehicle routing problem with time windows by means of ant colony system // IEEE Congress on Evolutionary Computation (CEC), 2017. P. 2480-2487. https://doi.org/10.1109/CEC.2017.7969606

21. Papadimitriou C. Euclidean TSP is NP-complete // Theoret. Comput. Sci. 1977. Vol. 4. P. 237-244.

22. Pecin D., Pessoa A., Poggi M., Uchoa E. Improved branch-cut-and-price for capacitated vehicle routing // Mathematical Programming Computation. 2017. Vol. 9, no. 1. P. 61-100. https://doi.org/10.1007/s12532-016-0108-8

23. Pessoa A. A., Sadykov R., Uchoa E. Enhanced branch-cut-and-price algorithm for heterogeneous fleet vehicle routing problems // European J. Oper. Res. 2018. Vol. 270, no. 2. P. 530-543. https://doi.org/10.1016/j.ejor.2018.04.009 
24. Pessoa A. A., Sadykov R., Uchoa E., Vanderbeck F. A generic exact solver for vehicle routing and related problems // Integer Programming and Combinatorial Optimization: Proc. 20th Inter. Conf. / eds. A. Lodi, V. Nagarajan. Cham: Springer, 2019, pp. 354-369. (Lecture Notes in Computer Science; vol. 11480). https://doi.org/10.1007/978-3-030-17953-3_27

25. Polat O. A parallel variable neighborhood search for the vehicle routing problem with divisible deliveries and pickups // Comput. Oper. Res. 2017. Vol. 85. P. 71-86. https://doi.org/10.1016/j.cor.2017.03.009

26. Qiu M., Fu Z., Eglese R., Tang Q. A tabu search algorithm for the vehicle routing problem with discrete split deliveries and pickups // Comput. Oper. Res. 2018. Vol. 100. P. 102-116. https://doi.org/10.1016/j.cor.2018.07.021

27. Toth P., Vigo D. Vehicle routing: Problems, methods and applications. Second Edition. MOS-Siam Series on Optimization, SIAM, 2 edn. 2014. 481 p.

28. Vidal T., Crainic T. G., Gendreau M., Prins C. A hybrid genetic algorithm with adaptive diversity management for a large class of vehicle routing problems with time-windows // Comput. Oper. Res. 2013. Vol. 40, no. 1. P. 475-489. https://doi.org/10.1016/j.cor.2012.07.018

Поступила 30.08.2019

После доработки 30.09.2019

Принята к публикации 7.10.2019

Хачай Михаил Юрьевич

д-р физ.-мат. наук

профессор РАH

зав. отделом

Институт математики и механики им. Н.Н. Красовского УрО РАН;

Уральский федеральный университет им. Б. Н. Ельцина

г. Екатеринбург

Омский государственный технический университет

г. Омск

e-mail: mkhachay@imm.uran.ru

Огородников Юрий Юрьевич

науч. сотрудник

Институт математики и механики им. Н. Н. Красовского УрО РАН;

Уральский федеральный университет им. Б. Н. Ельцина

г. Екатеринбург

e-mail: yogorodnikov@gmail.com

\section{REFERENCES}

1. Abraham I., Bartal Y., Neiman O. Advances in metric embedding theory. Advances in Mathematics, 2011, vol. 228, no. 6, pp. 3026-3126. https://doi.org/10.1016/j.aim.2011.08.003

2. Adamaszek A., Czumaj A., Lingas A. PTAS for $k$-tour cover problem on the plane for moderately large values of $k$. Inter. J. Foundations of Computer Science, 2010, vol. 21, no. 06, pp. 893-904. https://doi.org/10.1142/S0129054110007623

3. Arnold F., Sörensen K. Knowledge-guided local search for the vehicle routing problem. Comput. Oper. Res., 2019, vol. 105, pp. 32-46. https://doi.org/10.1016/j.cor.2019.01.002

4. Arora S. Polynomial time approximation schemes for Euclidean traveling salesman and other geometric problems. J. ACM, 1998, vol. 45, pp. 753-782.

5. Asano T., Katoh N., Tamaki H., Tokuyama T. Covering points in the plane by $k$-tours: Towards a polynomial time approximation scheme for general $k$. In: Proc. of the Twenty-ninth Annual ACM Symposium on Theory of Computing (STOC '97), N Y, USA: ACM, 1997. P. 275-283. https://doi.org/10.1145/258533.258602

6. Assouad P. Plongements lipschitziens dans $\mathbb{R}^{n}$. Bulletin de la Société Mathématique de France, 1983, vol. 111, pp. 429-448. http://eudml.org/doc/87452

7. Bartal Y., Gottlieb L. A., Krauthgamer R. The traveling salesman problem: Low-dimensionality implies a polynomial time approximation scheme. SIAM J. on Computing, 2016, vol. 45, no. 4, pp. 1563-1581. https://doi.org/10.1137/130913328 
8. Becker A., Klein P.N., Schild A. A PTAS for bounded-capacity vehicle routing in planar graphs. In: Friggstad Z., Sack J.-R., Salavatipour M. (eds), Algorithms and Data Structures, 2019, Lecture Notes in Computer Science, vol. 11646, Cham: Springer, pp. 99-111.

9. Dantzig G. B., Ramser J.H. The truck dispatching problem. Management science, 1959, vol. 6, no. 1, pp. $80-91$.

10. Das A., Mathieu C. A quasipolynomial time approximation scheme for Euclidean capacitated vehicle routing. Algorithmica, 2015, vol. 73, pp. 115-142. https://doi.org/10.1007/s00453-014-9906-4

11. Demir E., Huckle K., Syntetos A., Lahy A., Wilson M. Vehicle routing problem: Past and future. In: Wells P. (ed.), Contemporary Operations and Logistics, Cham: Springer, 2019, pp. 97-117. https://doi.org/10.1007/978-3-030-14493-7 7

12. Haimovich M., Rinnooy Kan A.H. G. Bounds and heuristics for capacitated routing problems. Math. Oper. Res., 1985, vol. 10, no. 4, pp. 527-542. https://doi.org/10.1287/moor.10.4.527

13. van Hoorn J. J. Dynamic programming for routing and scheduling: Optimizing sequences of decisions. Ph. D. thesis, 2016, 210 p. https://doi.org/10.13140/RG.2.2.14344.88329

14. Khachay M., Ogorodnikov Y. Efficient PTAS for the Euclidean CVRP with time windows. In: van der Aalst W. et al. (eds), Analysis of Images, Social Networks and Texts (AIST 2018), Lecture Notes in Computer Science, vol. 11179, Cham: Springer, 2018, pp. 318-328.

https://doi.org/10.1007/978-3-030-11027-7_30

15. Khachay M., Ogorodnikov Y. Approximation scheme for the capacitated vehicle routing problem with time windows and non-uniform demand. In: Khachay M., Kochetov Y., Pardalos P. (eds), Mathematical Optimization Theory and Operations Research (MOTOR 2019), Lecture Notes in Computer Science, vol. 11548, Cham: Springer, 2019, pp. 309-327. https://doi.org/10.1007/978-3-030-22629-9_22

16. Khachay M., Ogorodnikov Y. Improved polynomial time approximation scheme for capacitated vehicle routing problem with time windows. In: Evtushenko Y., Jaćimović M., Khachay M., Kochetov Y., Malkova V., Posypkin M. (eds), Optimization and Applications (OPTIMA 2018), Communications in Computer and Information Science, vol. 974, Cham: Springer, 2019, pp. 155-169.

https://doi.org/10.1007/978-3-030-10934-9_12

17. Khachay M., Dubinin R. PTAS for the Euclidean capacitated vehicle routing problem in $R^{d}$. In: Kochetov Y., Khachay M., Beresnev V., Nurminski E., Pardalos P. (eds), Discrete Optimization and Operations Research, Lecture Notes in Computer Science, vol. 9869, Cham: Springer, 2016, pp. 193-205. https://doi.org/10.1007/978-3-319-44914-2_16

18. Khachay M., Zaytseva H. Polynomial time approximation scheme for single-depot Euclidean capacitated vehicle routing problem. In: Lu Z., Kim D., Wu W., Li W., Du D.Z. (eds), Combinatorial Optimization and Applications, Lecture Notes in Computer Science, vol. 9486, Cham: Springer, 2015, pp. 178-190. https://doi.org/10.1007/978-3-319-26626-8_14

19. Nalepa J., Blocho M. Adaptive memetic algorithm for minimizing distance in the vehicle routing problem with time windows. Soft Computing, 2016, vol. 20, no. 6, pp. 2309-2327.

https://doi.org/10.1007/s00500-015-1642-4

20. Necula R., Breaban M., Raschip M. Tackling dynamic vehicle routing problem with time windows by means of ant colony system. IEEE Congress on Evolutionary Computation (CEC), 2017, pp. 2480-2487. https://doi.org/10.1109/CEC.2017.7969606

21. Papadimitriou C. Euclidean TSP is NP-complete Theoret. Comput. Sci., 1977, vol. 4, pp. 237-244.

22. Pecin D., Pessoa A., Poggi M., Uchoa E. Improved branch-cut-and-price for capacitated vehicle routing. Math. Programming Computation, 2017, vol. 9, no. 1, pp. 61-100.

https://doi.org/10.1007/s12532-016-0108-8

23. Pessoa A. A., Sadykov R., Uchoa E. Enhanced branch-cut-and-price algorithm for heterogeneous fleet vehicle routing problems. European J. Oper. Res., 2018, vol. 270, no. 2, pp. 530-543.

https://doi.org/10.1016/j.ejor.2018.04.009

24. Pessoa A.A., Sadykov R., Uchoa E., Vanderbeck F. A generic exact solver for vehicle routing and related problems. In: Lodi A., Nagarajan V. (eds), Proc. 20th Inter. Conf. "Integer Programming and Combinatorial Optimization" (IPCO), Lecture Notes in Computer Science, vol. 11480, Cham: Springer, 2019, pp. 354-369. https://doi.org/10.1007/978-3-030-17953-3_27

25. Polat $\mathrm{O}$. A parallel variable neighborhood search for the vehicle routing problem with divisible deliveries and pickups. Comput. Oper. Res., 2017, vol. 85, pp. 71-86. https://doi.org/10.1016/j.cor.2017.03.009

26. Qiu M., Fu Z., Eglese R., Tang Q. A tabu search algorithm for the vehicle routing problem with discrete split deliveries and pickups. Comput. Oper. Res., 2018, vol. 100, pp. 102-116.

https://doi.org/10.1016/j.cor.2018.07.021 
27. Toth P., Vigo D. Vehicle routing: Problems, methods and applications. Philadelphia: SIAM, 2014,481 p.

28. Vidal T., Crainic T. G., Gendreau M., Prins C. A hybrid genetic algorithm with adaptive diversity management for a large class of vehicle routing problems with time-windows. Comput. Oper. Res., 2013, vol. 40, no. 1, pp. 475-489. https://doi.org/10.1016/j.cor.2012.07.018

Received August 30, 2019

Revised September 30, 2019

Accepted October 7, 2019

Funding Agency: This research is supported by the Russian Foundation for Basic Research (projects no. 19-07-01243 and 17-08-01385).

Mikhail Yur'evich Khachai, Dr. Phys.-Math. Sci., Prof., Krasovskii Institute of Mathematics and Mechanics of the Ural Branch of the Russian Academy of Sciences, Yekaterinburg, 620108 Russia; Ural Federal University, Yekaterinburg, 620083 Russia; Omsk State Technical University, Omsk, 644050 Russia, e-mail: mkhachay@imm.uran.ru .

Yurii Yur'evich Ogorodnikov, Krasovskii Institute of Mathematics and Mechanics of the Ural Branch of the Russian Academy of Sciences, Yekaterinburg, 620108 Russia, Ural Federal University, Yekaterinburg, 6200083 Russia, e-mail: yogorodnikov@gmail.com .

Cite this article as: M. Yu. Khachai, Yu. Yu. Ogorodnikov. Haimovich-Rinnooy Kan polynomial-time approximation scheme for the CVRP in metric spaces of a fixed doubling dimension, Trudy Instituta Matematiki i Mekhaniki URO RAN, 2019, vol. 25, no. 4, pp. 235-248. 